\title{
Relationship between perfusion index and patent ductus arteriosus in preterm infants
}

\author{
Enrique Gomez-Pomar' ${ }^{1}$ Majd Makhoul'2, Philip M. Westgate ${ }^{3}$, Katrina T. Ibonia' , Abhijit Patwardhan4, Peter J. Giannone', \\ Henrietta S Bada' and Elie G. Abu Jawdeh'
}

BACKGROUND: Perfusion index (PI) is a noninvasive measure of perfusion. $\triangle \mathrm{PI}$ (difference between pre- and postductal PI) may identify hemodynamically significant PDA. However, studies are limited to brief and intermittent $\triangle \mathrm{PI}$ sampling. Our objective is to assess the value of continuous high resolution $\triangle \mathrm{PI}$ monitoring in the diagnosis of PDA.

METHODS: Continuous $\triangle \mathrm{PI}$ monitoring in preterm infants was prospectively performed using two high-resolution pulse oximeters. Perfusion Index measures ( $\triangle \mathrm{PI}$ mean and variability, pre- and postductal PI) were analyzed over a 4-h period prior to echocardiography. A cardiologist blinded to the results evaluated for PDA on echocardiography. Linear mixed regression models were utilized for analyses.

RESULTS: We obtained 31 echocardiography observations. Mean $\triangle \mathrm{PI}(-0.23$ vs. $0.16 ; P<0.05)$, mean pre-PI $(0.86$ vs. $1.26 ; P$ $<0.05)$, and $\triangle P$ I variability ( 0.39 vs. $0.61 ; P=0.05$ ) were lower in infants with PDA compared to infants without PDA at the time of echocardiography.

CONCLUSION: Mean $\triangle \mathrm{PI}, \triangle \mathrm{PI}$ variability, and mean pre-PI measured $4 \mathrm{~h}$ prior to echocardiography detect PDA in preterm infants. PI is dynamic and should be assessed continuously. Perfusion index is a promising bedside measurement to identify PDA in preterm infants.

$\mathbf{P}$ atent ductus arteriosus (PDA), a common condition in preterm infants, leads to shunting of blood between the systemic and the pulmonary circulations. Approximately $65 \%$ of infants born between 25 and 28 wk gestational age (GA), and $85 \%$ of those born at $24 \mathrm{wk}$ GA will have PDA at first week of life (1). Persistent patency is associated with adverse outcomes, including prolonged assisted ventilation and higher rates of death, bronchopulmonary dysplasia, pulmonary hemorrhage, necrotizing enterocolitis, impaired renal function, intraventricular hemorrhage, periventricular leukomalacia, and cerebral palsy $(1,2)$. Because of these associated complications, majority of infants $<28 \mathrm{wk}$ GA will receive medical or surgical therapy in an attempt to close the PDA (1-3). Currently, the gold standard for PDA diagnosis is echocardiography $(2,4-8)$, and often clinical symptoms are not associated with echocardiography findings $(1,4)$.

Perfusion index (PI) is a noninvasive measure for monitoring the general hemodynamic status of the preterm infant (9-11). Perfusion index provides assessment for the pulse strength and is derived from pulse oximetry. PI, measured by infrared light, is calculated as the ratio of the pulsatile (AC) to nonpulsatile components (DC) of the blood flow in tissue $(9,10,12,13)$. In neonates, PI has clinical application. Granelli et al. (14) correlated lower PI values in infants with critical left heart obstructive disease. In addition, De Felice et al. (15) reported that PI was decreased in infants born to mothers with chorioamnionitis.

Reports are inconsistent as to the value of PI in the assessment of PDA. This may be attributed in part to location and the duration of PI measurements $(12,16)$. Khositseth et al. (16) hypothesized that the peripheral perfusion of the lower extremities (postductal) is decreased compared to the right arm (preductal) in preterm infants with hemodynamically significant patent ductus arteriosus (hsPDA). This difference is due to left-to-right shunt across the ductus arteriosus into the pulmonary artery. They reported that a difference in PI between the upper and the lower extremity, or delta PI $(\Delta \mathrm{PI})$, of more than $1.05 \%$ strongly correlated with the echocardiographic diagnosis of hsPDA (sensitivity: $66.7 \%$, specificity: $100 \%$, positive predictive value: $100 \%$ and negative predictive value: $86.4 \%$ ). Their study was limited by a one-time observation that may not be reflective of the hemodynamic variability of perfusion in infants with PDA. Alternatively, Vidal et al. (12) conducted a study to evaluate the postductal PI of premature infants in order to categorize the PDA status and found that postductal PI did not correlate with PDA and was not influenced by ductal flow pattern.

We conducted a prospective study to assess the value of $\Delta \mathrm{PI}$ in the diagnosis of PDA in preterm infants, using high resolution continuous pre- and postductal monitoring.

\footnotetext{
'Division of Neonatology, Department of Pediatrics, University of Kentucky, Lexington, Kentucky; ${ }^{2}$ Division of Cardiology, Department of Pediatrics, University of Kentucky, Lexington, Kentucky; ${ }^{3}$ Department of Biostatistics, University of Kentucky, Lexington, Kentucky; ${ }^{4}$ Biomedical Engineering, University of Kentucky, Lexington, Kentucky. Correspondence: Enrique Gomez-Pomar (Enrique.gomez@uky.edu)

Received 14 June 2016; accepted 27 December 2016; advance online publication 5 April 2017. doi:10.1038/pr.2017.10
} 


\section{METHODS}

This prospective study was conducted at a level IV NICU between November 2014 and July 2015. The study was approved by the Institutional Review Board of the University of Kentucky and parental informed consent was obtained in all cases. Infants with GA $\leq 29 \mathrm{wk}$ were enrolled on the first day of life and followed for a 2-wk period. Infants with major congenital malformations were excluded. Those infants in which we had an echocardiography and adequate PI data for $4 \mathrm{~h}$ prior to the echocardiography were chosen for analysis.

\section{Perfusion Index Measurement}

Perfusion index was continuously monitored using high resolution ( $2 \mathrm{~s}$ averaging time, $1 \mathrm{~Hz}$ sampling rate) pulse oximeters (Masimo Radical-Masimo Corporation, Irvine, CA). In order to capture echocardiograms performed for PDA assessment, data were recorded continuously during the first $14 \mathrm{~d}$ of life. Subjects were connected to two pulse oximeters simultaneously; right upper extremity for preductal monitoring and either lower extremity for postductal monitoring. Data from pulse oximeters were continuously stored to serial data recorders. The pre- and postductal PI difference $(\Delta \mathrm{PI})$ was defined as the PI measured preductal minus the PI measured postductal (16).

\section{Echocardiography}

Two-dimensional, color Doppler, spectral Doppler, and M-mode echocardiography was performed to assess for PDA at the discretion of the attending physician using a Phillips IE33 echocardiography machine with $12-\mathrm{MHz}$ transducer. A cardiologist, blinded to the results of the study, independently examined the echo images and categorized subjects into the following three groups: (i) hemodynamically significant PDA (hsPDA); (ii) nonhemodynamically significant PDA (non-hsPDA), and (iii) no PDA. The definition of hsPDA included infants with at least two of the following: (i) ductal diameter at the pulmonary side $\geq 1.4 \mathrm{~mm} / \mathrm{kg}$; (ii) left atrial to aortic ratio $\geq 1.5$; (iii) left pulmonary artery (LPA) mean flow velocity of $\geq 0.42 \mathrm{~m} / \mathrm{s}$; and (iv) LPA end-diastolic velocity of $\geq 0.2 \mathrm{~m} / \mathrm{s}(3,5,7,17-19)$.

\section{Sample Size}

In order to determine the minimum sample size needed to assess the value of $\triangle \mathrm{PI}$ in PDA diagnosis, we utilized the results reported in the pilot data by Khositseth et al. (16). Assuming the $\Delta$ PI (\%) mean and SD are 1.00 and 0.70 , respectively, for children with PDA and 0.04 and 0.10 , respectively, for children with no PDA, we calculated a total required study sample size of 15 infants (power $80 \%, \alpha=0.05$ ).

\section{Data Management and Statistical Analysis}

The pulse oximeters serial data recorders were time synced. Perfusion Index sampling rate was $1 \mathrm{~Hz}$ (every second). However, there were rare occurrences of two values per second. In such cases, the average value for the given second was utilized. In order to better visualize an example of PI values over time (Figure 2), we plot PI values that were averaged over each minute. Any given value, at any given second, by itself will not represent a true overall reflection of PI for the duration of several hours, and thus cannot be used to accurately predict PDA. We therefore decided, for predictive purposes, to assess the utility of average $\Delta \mathrm{PI}$ values during the $4 \mathrm{~h}$ prior to an echocardiography as a single measure of PI to predict PDA which could better represent the hemodynamic status of preterm infants. This period of $4 \mathrm{~h}$ will capture changes resulting from the ultradian rhythm that has been reported in premature infants (20). Subjects with $4 \mathrm{~h}$ of adequate monitoring prior to the echocardiography were considered for analysis. Artifacts and extreme values, found in less than $2 \%$ of PI measurements, were removed as they were associated with inadequate signal capture.

Data analyses were conducted by a statistician. The primary outcome of interest is the average $\Delta \mathrm{PI}$ during the $4 \mathrm{~h}$ leading up to echocardiography and pre- and postductal PI were secondary outcomes. Furthermore, PI variability was analyzed by using the outcome of the $\mathrm{SD}$ of the individual PI values over the $4 \mathrm{~h}$. When comparing mean values for no PDA, non-hsPDA and hsPDA, linear mixed regression models were utilized in order to account for repeated measurements in subjects with multiple echocardiograms. The Kenward and Roger degrees of freedom method was used for inference (21). Generalized estimating equations with the Kauermann and Carroll correction (22) and between-within degrees of freedom were used to evaluate baseline differences among groups defined by PDA status. Analyses were conducted in SAS Version 9.4 (SAS Institute, Cary, NC). All tests were two-sided at the $5 \%$ significance level.

\section{RESULTS}

A total of 40 infants were enrolled upon admission. Of these, 4 had no echocardiography performed and 16 had missing PI data or artifacts during the study period. Final analyses included data from 20 infants with a total of 31 echocardiography observations (each infant was observed at 1 to 3 occasions) (Figure 1). Eighteen infants were found to have PDA on echocardiography. The characteristics at birth of the infants did not significantly differ between those with and without PDA, as shown in Table 1 . The baseline characteristics of the infants at the time of echocardiography are presented in Table 2; no statistically significant differences were noted among groups. As represented in Figure 2, PI values were found to be highly variable with changes every minute.

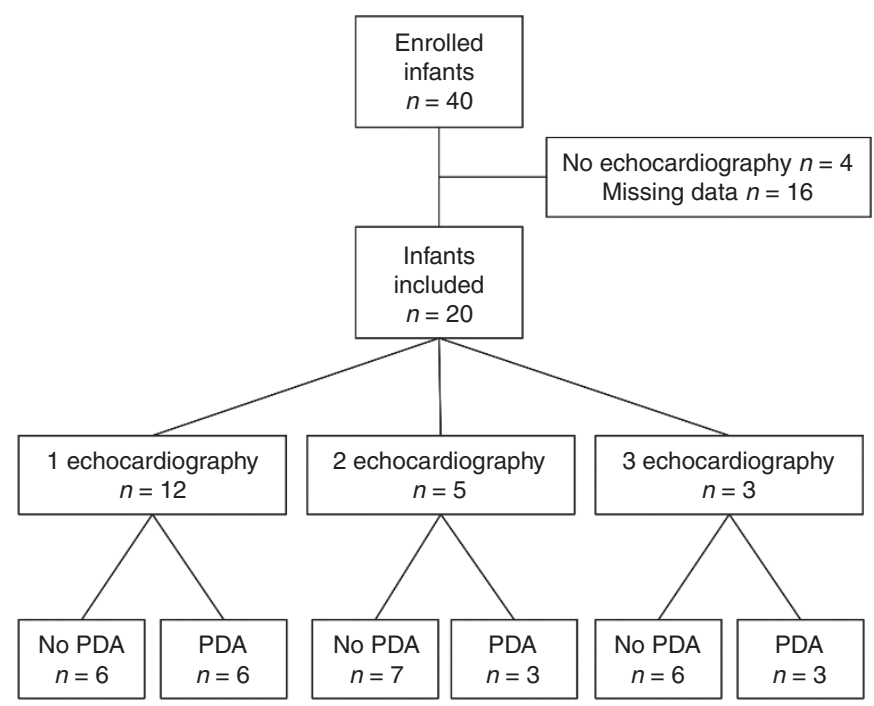

Figure 1. Flow diagram of the enrolled patients.

Table 1. Characteristics of infants at birth*

\begin{tabular}{lcc}
\hline & PDA $\boldsymbol{n}=\mathbf{1 8}$ & No PDA $\boldsymbol{n}=\mathbf{1 3}$ \\
\hline GA weeks, median (IQR) & $264 / 7(252 / 7,276 / 7)$ & $252 / 7(251 / 7,263 / 7)$ \\
Sex, male (\%) & 83 & 46 \\
Weight, median (IQR) & $931(780,1,030)$ & $730(660,855)$ \\
Antenatal steroids (\%) & 89 & 77 \\
Chorioamnionitis (\%) & 6 & 15 \\
Caesarean section (\%) & 61 & 62 \\
Surfactant in delivery & 67 & 77 \\
room (\%) & & $12(8,15)$ \\
Time (minutes) to first & $9(7,96)$ & $3(2-8)$ \\
surfactant, median (IQR) & & $6(2-8)$ \\
Apgar 1, median (range) & $4(1-8)$ & \\
Apgar 5, median (range) & $6(1-5)$ & \\
\hline
\end{tabular}

* $\mathrm{QQR}$, interquartile range.

All $P=$ NS by Wilcoxon-Mann-Whitney test or chi-square. 
Table 2. Infant characteristics at the time of echocardiography, comparing groups, and subgroups*

\begin{tabular}{|c|c|c|c|c|}
\hline & No PDA & All PDA & hsPDA & Non-hsPDA \\
\hline$N$ & 13 & 18 & 7 & 11 \\
\hline Day of life, median (IQR) & $8(5,11)$ & $7(2,6)$ & $6(5,7)$ & $8(6,9)$ \\
\hline Weight, median (IQR) & $730(660,855)$ & $931(780,1030)$ & $1,021(905,1030)$ & $880(775,1,005)$ \\
\hline Heart rate, median (IQR) & $163(144,173)$ & $165(156,172)$ & $165(157,173)$ & $164(156,168)$ \\
\hline Systolic blood pressure, median (IQR) & $56.0(47.0,66.0)$ & $62.0(51.5,74.0)$ & $60.0(53.0,73.0)$ & $63.0(50.5,75.5)$ \\
\hline Diastolic blood pressure, median (IQR) & $28.0(24.0,45.0)$ & $36.0(30.0,43.7)$ & $43.0(36.5,43.5)$ & $34.0(26.0,43.0)$ \\
\hline Mean blood pressure, median (IQR) & $42.0(35.0,48.0)$ & $41.5(39.2,48.2)$ & $42.0(41.0,50.0)$ & $40.0(35.5,42.5)$ \\
\hline$\%$ infants on mechanical ventilation & $92 \%$ & $77 \%$ & $71 \%$ & $81 \%$ \\
\hline $\mathrm{pH}$ & $7.3(7.3,7.4)$ & $7.3(7.3,7.4)$ & $7.3(7.3,7.4)$ & $7.3(7.3,7.4)$ \\
\hline pCO2 & $43.0(36.0,55.0)$ & $44.5(35.0,52.2)$ & $42.0(32.0,47.5)$ & $45.0(36.0,53.0)$ \\
\hline $\mathrm{HCO} 3$ & $28.0(22.0,29.0)$ & $23.0(20.2,25.7)$ & $23.0(19.5,24.0)$ & $24.0(21.5,26.5)$ \\
\hline Base deficit & $3.5(2.1,5.5)$ & $3.8(2.2,6.3)$ & $3.9(2.5,5.9)$ & $3.5(2.3,6.5)$ \\
\hline Urine output ( $24 \mathrm{~h}$ ), median (IQR) & $3.26(2.71,3.41)$ & $2.75(1.97,3.90)$ & $3.70(1.90,4.76)$ & $2.70(2.18,3.39)$ \\
\hline Creatinine, median (IQR) & $0.78(0.66,0.88)$ & $0.71(0.66,0.88)$ & $0.71(0.66,0.81)$ & $0.70(0.67,0.88)$ \\
\hline Total fluid volume/kg/day, median (IQR) & $135(130,150)$ & $130(122,140)$ & $130(125,135)$ & $140(125,145)$ \\
\hline
\end{tabular}

IQR, interquartile range; $P E E P$, positive end-expiratory pressure.

All $P=$ NS by Wilcoxon-Mann-Whitney test or chi-square.

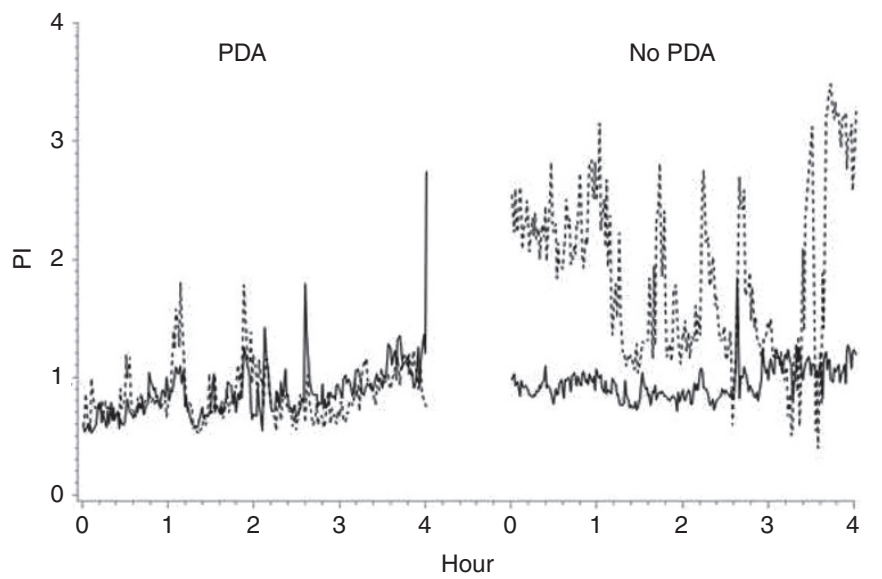

Figure 2. Sample plot representing PI values of one infant with PDA (Variability 0.33 , Mean -0.01 ) and no PDA (Variability 0.71 , Mean 0.87 ) for $4 \mathrm{~h}$ prior to an echocardiogram. Dashed lines represent the preductal PI and solid lines represent the postductal PI.

Mean $\triangle$ PI differed significantly between infants with PDA and without PDA (Figure 3). Mean pre- and postductal PI values are presented in Figure 4. The preductal PI was significantly elevated in infants without PDA as compared to infants with PDA. Among the PDA subgroups, the preductal PI of those with nonhsPDA was lower compared to infants without PDA (Figure 4). The mean postductal PI did not differ among groups (Figure 4).

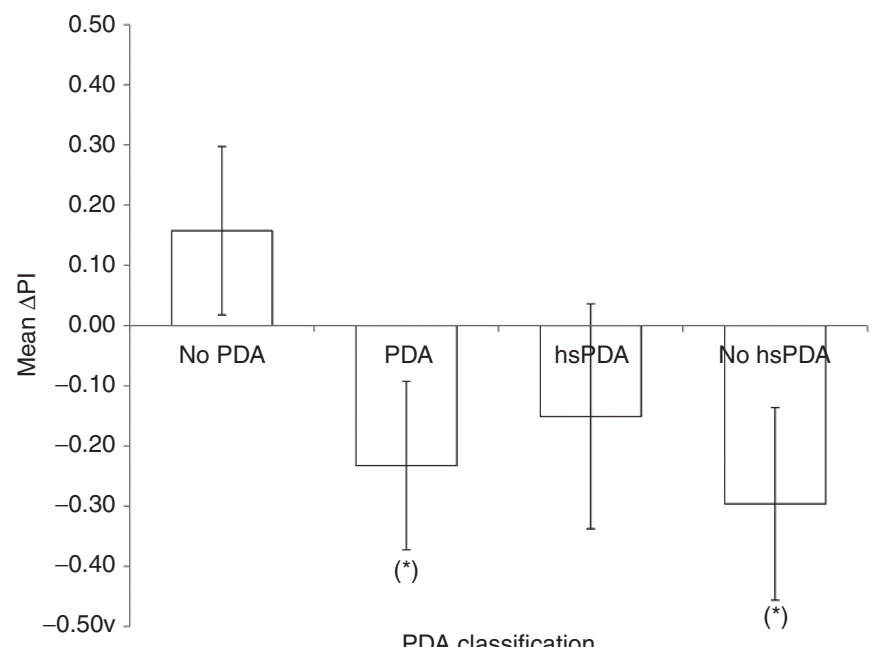

Figure 3. Mean \pm SD values of Delta Perfusion Index (ÄPI) $4 \mathrm{~h}$ prior to echocardiography. Comparing ÄPI in infants with no PDA vs. infants with PDA, hemodynamically significant PDA (hsPDA) and no hsPDA. ${ }^{*} P<0.05$ compared to no PDA.

Variability of $\Delta \mathrm{PI}$, pre- and postductal PI is presented in Figure 5. $\triangle \mathrm{PI}$ variability was significantly lower in infants with PDA compared to no PDA. Although not statistically significant, the PI variability is consistently low in infants with PDA for pre- and postductal measures. 


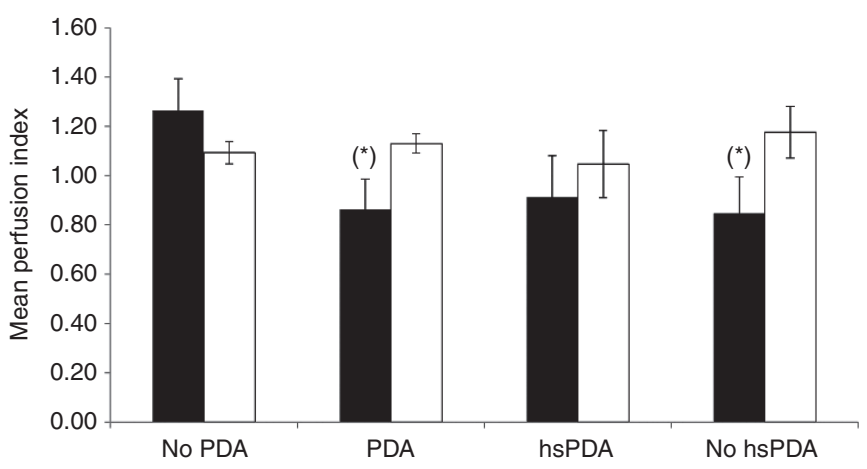

Figure 4. Mean \pm SD values of Perfusion Index (PI) $4 \mathrm{~h}$ prior to echocardiography. Comparing the preductal (black bar) and postductal (white bar) PI of infants with no PDA vs. infants with PDA, hemodynamically significant PDA (hsPDA) and no hsPDA. ${ }^{*} P<0.05$ compared to no PDA.

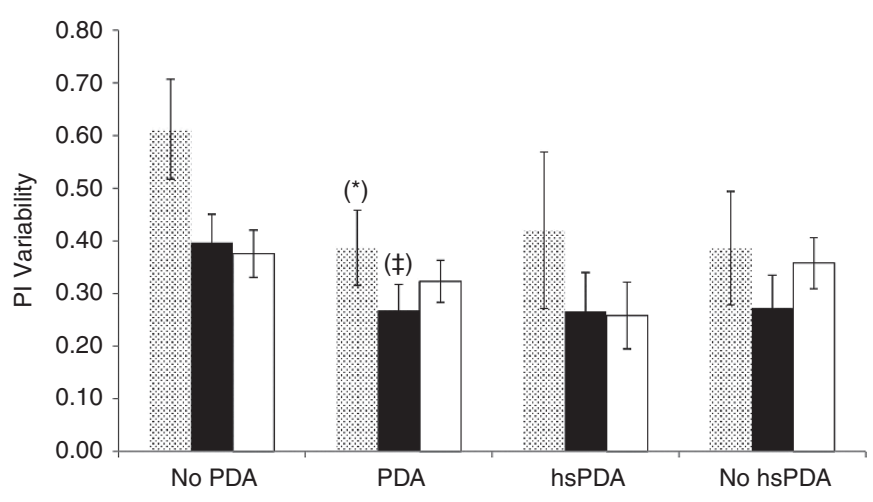

Figure 5. Mean \pm SD values of the Variability of Perfusion Index (PI) $4 \mathrm{~h}$ prior to echocardiography. Comparing $\triangle \mathrm{PI}$ (dotted bar), preductal PI (black bar) and postductal PI (white bar) variability for infants with no PDA vs. with PDA, hemodynamically significant PDA (hsPDA) and no hsPDA. ${ }^{*} P<0.05$ and ${ }^{\ddagger} P=0.08$ compared to no PDA.

\section{DISCUSSION}

Our study demonstrates that the mean $\Delta \mathrm{PI}$, mean pre-PI and the $\Delta$ PI variability can identify PDA in premature infants. Mean values of $\Delta \mathrm{PI}$, pre- and postductal PI and $\Delta \mathrm{PI}$ variability were continuously calculated over the 4 -h period prior to echocardiography compared to intermittent measures as previously described $(11,12,14,23,24)$. Our observations are somewhat contradictory to initial expectations related to changes in preductal PI and $\Delta$ PI. We expected to observe a steady preductal PI and a decreased postductal PI leading to a larger $\Delta \mathrm{PI}$ in infants with PDA. The negative $\triangle \mathrm{PI}$ (Figure 3 ) is likely a combination of a decreased preductal PI (reported by Karadag et al. (25)) and a postductal PI that is either steady (reported by Vidal et al. (12)) or increased (reported by Alderliesten $e$ t al. (9)). These results have a combined effect towards a negative $\Delta$ PI value found in infants with PDA.

We found the preductal PI to be significantly lower in infants with PDA compared to infants without PDA (Figure 4). To understand this result, we refer to the definition of PI (AC/ $\left.\mathrm{DC}^{\star} 100\right)(15,26)$, wherein $\mathrm{AC}$ is the pulsatile component of the signal and DC is the nonpulsatile component. Infants with PDA can have an absent or reverse flow during diastole in the postductal sites but continuous forward blood flow in the preductal sites $(7,8)$. In infants with PDA, there is also an increase in the cardiac output to compensate for the decreased perfusion in the postductal sites (27-29). This change in cardiac output increases the preductal DC component in infants with PDA compared to no PDA; explaining why the preductal PI is lower in these infants. Our results are consistent with Karadag et al. (25) who analyzed the preductal PI in infants treated with surfactant. They found that the incidence of PDA was greater among the infants with a lower preductal PI.

Our study shows no difference between mean postductal PI in infants with PDA and no PDA. Our findings are consistent with Vidal et al. (12) who found no statistically significant difference or correlation between postductal PI and PDA in premature infants. Although not statistically significant, the postductal PI was higher in our infants with PDA compared to infants without PDA (Figure 4). We believe that with PDA there is a decrease in the DC component of the postductal PI due to the overall lower perfusion and decreased mean arterial pressure at the postductal sites $(30,31)$. Furthermore, our findings are consistent with the report by Alderliesten et al. (9) who found in a study of 342 neonates that infants with hsPDA had higher postductal PI than infants without hsPDA. They attributed this finding to a hyperdynamic circulation with a widened pulse-pressure resulting in an increase in the AC component. We believe that the increase in postductal PI, if present, is the result of a combination of the effect of the elevated AC component (due to the hyperdynamic circulation) and a decreased DC component (due to a decreased general perfusion).

Given that the mean $\Delta$ PI may not reflect instantaneous hemodynamic changes, we also assessed the variability of the $\Delta \mathrm{PI}$ over the 4-h monitoring period. Since the correlation of blood flow and PI has already been established $(11,32)$, we believe that the $\Delta \mathrm{PI}$ variability should also correlate with the hemodynamic status of the infant. Our findings show that infants with PDA have significantly lower $\triangle \mathrm{PI}$ variability compared to those with no PDA (Figure 5). Although trending in the same direction, changes in variability were not statistically significant for pre- and postductal PI (Figure 5). The change in $\Delta \mathrm{PI}$ variability observed in our study is noteworthy since it has not been previously described. De Felice et al. (15) speculated that changes in PI variability may be associated with neonatal morbidities, similar to heart rate variability. Decreased heart rate variability in preterm infants with PDA was described by Prietsch et al. (33). This decreased heart rate variability resolved after treatment with indomethacin. $\Delta \mathrm{PI}$ and heart rate variability are valuable at identifying subclinical cardiovascular dysfunction in preterm infants (15). The variable PI, as a reflection of the changing hemodynamic status of infants, may also explain the discrepancy among PI values reported in different studies $(9,13,14,23,24)$. Compared to other studies $(11,12,14,23,24)$, we measured PI with high resolution ( $1 \mathrm{~s}$ sampling rate) continuous pulse oximetry which gives our study the strength of having high quality monitoring for long periods of time. We advocate for continuous measurement of PI compared to spot checks; however, the question that remains to be answered is the optimal monitoring duration needed to detect hemodynamic instability. 
The echocardiographic classification of hsPDA used in this study is commonly reported in the literature $(8,30,31)$ but did not correspond to the clinical status of our infants. Those infants designated by echocardiography as hsPDA required less mechanical ventilation, had less $\mathrm{FiO} 2$ requirement, and no difference in acidosis compared to non-hsPDA; although not statistically significant (Table 2). It is possible that mechanical ventilation may have an effect on PI measures; however, our sample size does not allow to determine an independent effect of ventilation on PI changes. Our study was not designed to establish any correlation between the PI values and the clinical severity of the ductus arteriosus. Even though the ductal stealing phenomenon in infants with PDA is well known $(17,30,31)$, its relationship with end organ hypoperfusion and neonatal morbidity remains controversial (34).

Although we achieved the planned observations per our power calculation (accounting for data loss), our sample size is small to evaluate other factors that may affect PI values. Our study has the strength of offering continuous high-quality monitoring throughout the study period. This allowed us to adequately assess the relationship between PI and PDA.

We were able to demonstrate that a lower mean $\Delta \mathrm{PI}$ and pre PI values over a 4 -h period have the potential to detect the presence of PDA in premature infants. We are the first to report a lower variability in $\triangle \mathrm{PI}$ in infants with PDA compared to infants without PDA. Perfusion index provided by the bedside monitor is a promising bedside tool to assess for PDA in preterm infants. Future studies with a large cohort are needed to determine the clinical utility of PI in predicting PDA and monitoring of its hemodynamic course through days of treatment.

\section{ACKNOWLEDGMENTS}

The authors are thankful to the NICU faculty, nurses, research staff, and families.

\section{STATEMENT OF FINANCIAL SUPPORT}

The study was funded by National Center for Research Resources, UL1RR033173, and is now at the National Center for Advancing Translational Sciences (E.G., E.G.A.J.); and The Gerber Foundation (E.G.A.J., P.W., P.G., H.B.).

Disclosure: The authors have no financial relationships relevant to this article to disclose. The authors have no conflicts of interest to disclose.

\section{REFERENCES}

1. Benitz WE, Committee On Fetus and Newborn, 2016 Patent Ductus Arteriosus in Preterm Infants. Pediatrics 137:1-6.

2. Dice JE, Bhatia J. Patent ductus arteriosus: an overview. J Pediatr Pharmacol Ther 2007;12:138-46.

3. Hamrick SE, Hansmann G. Patent ductus arteriosus of the preterm infant. Pediatrics 2010;125:1020-30.

4. Skelton R, Evans N, Smythe J. A blinded comparison of clinical and echocardiographic evaluation of the preterm infant for patent ductus arteriosus. J Paediatr Child Health 1994;30:406-11.

5. Visconti LF, Morhy SS, Deutsch AD, Tavares GM, Wilberg TJ, Rossi Fde S. Clinical and echocardiographic characteristics associated with the evolution of the ductus arteriosus in the neonate with birth weight lower than 1,500g. Einstein (Sao Paulo) 2013;11:317-23.

6. Zonnenberg I, de Waal $\mathrm{K}$. The definition of a haemodynamic significant duct in randomized controlled trials: a systematic literature review. Acta Paediatr 2012;101:247-51.

7. Evans N, Malcolm G, Osborn D, Kluckow M 2004 Diagnosis of patent ductus arteriosus in preterm infants. NeoReviews 5:e86-e97.

8. Evans N. Diagnosis of the preterm patent ductus arteriosus: clinical signs, biomarkers, or ultrasound? Semin Perinatol 2012;36:114-22.
9. Alderliesten T, Lemmers PM, Baerts W, Groenendaal F, van Bel F. Perfusion index in preterm infants during the first 3 days of life: reference values and relation with clinical variables. Neonatology 2015;107:258-65.

10. Piasek CZ, Van Bel F, Sola A. Perfusion index in newborn infants: a noninvasive tool for neonatal monitoring. Acta Paediatr 2014;103:468-73.

11. Zaramella P, Freato F, Quaresima V, et al. Foot pulse oximeter perfusion index correlates with calf muscle perfusion measured by near-infrared spectroscopy in healthy neonates. J Perinatol 2005;25:417-22.

12. Vidal M, Ferragu F, Durand S, Baleine J, Batista-Novais AR, Cambonie G. Perfusion index and its dynamic changes in preterm neonates with patent ductus arteriosus. Acta Paediatr 2013;102:373-8.

13. Kinoshita M, Hawkes CP, Ryan CA, Dempsey EM. Perfusion index in the very preterm infant. Acta Paediatr 2013;102:e398-401.

14. Granelli Ad, Ostman-Smith I. Noninvasive peripheral perfusion index as a possible tool for screening for critical left heart obstruction. Acta Paediatr 2007;96:1455-9.

15. De Felice C, Goldstein MR, Parrini S, Verrotti A, Criscuolo M, Latini G. Early dynamic changes in pulse oximetry signals in preterm newborns with histologic chorioamnionitis. Pediatr Crit Care Med 2006;7:138-42.

16. Khositseth A, Muangyod N, Nuntnarumit P. Perfusion index as a diagnostic tool for patent ductus arteriosus in preterm infants. Neonatology 2013;104:250-4.

17. Sehgal A, McNamara PJ. Does echocardiography facilitate determination of hemodynamic significance attributable to the ductus arteriosus? Eur J Pediatr 2009;168:907-14.

18. Sehgal A, Paul E, Menahem S. Functional echocardiography in staging for ductal disease severity: role in predicting outcomes. Eur J Pediatr 2013;172:179-84.

19. El Hajjar M, Vaksmann G, Rakza T, Kongolo G, Storme L 2005 Severity of the ductal shunt: a comparison of different markers. Arch Dis Child Fetal Neonatal Ed 90:F419-422.

20. Shimada M, Takahashi K, Segawa M, Higurashi M, Samejim M, Horiuchi K. Emerging and entraining patterns of the sleep-wake rhythm in preterm and term infants. Brain Dev 1999;21:468-73.

21. Kenward MG, Roger JH. Small sample inference for fixed effects from restricted maximum likelihood. Biometrics 1997;53:983-97.

22. Liang K-Y, Zeger SL 1986 Longitudinal data analysis using generalized linear models. Biometrika 73:13-22.

23. Cresi F, Pelle E, Calabrese R, Costa L, Farinasso D, Silvestro L. Perfusion index variations in clinically and hemodynamically stable preterm newborns in the first week of life. Ital J Pediatr 2010;36:6.

24. Hakan N, Dilli D, Zenciroglu A, Aydin M, Okumus N. Reference values of perfusion indices in hemodynamically stable newborns during the early neonatal period. Eur J Pediatr 2014;173:597-602.

25. Karadag N, Dilli D, Zenciroglu A, Aydin B, Beken S, Okumus N. Perfusion index variability in preterm infants treated with two different natural surfactants for respiratory distress syndrome. Am J Perinatol 2014;31:101522.

26. De Felice C, Latini G, Vacca P, Kopotic RJ. The pulse oximeter perfusion index as a predictor for high illness severity in neonates. Eur J Pediatr 2002;161:561-2.

27. Evans N, Iyer P. Change in blood pressure after treatment of patent ductus arteriosus with indomethacin. Arch Dis Child 1993;68(5 Spec No): 584-7.

28. Freeman-Ladd M, Cohen JB, Carver JD, Huhta JC. The hemodynamic effects of neonatal patent ductus arteriosus shunting on superior mesenteric artery blood flow. J Perinatol 2005;25:459-62.

29. Shimada S, Kasai T, Hoshi A, Murata A, Chida S. Cardiocirculatory effects of patent ductus arteriosus in extremely low-birth-weight infants with respiratory distress syndrome. Pediatr Int 2003;45:255-62.

30. Volpe JJ, Perlman JM, Hill A, McMenamin JB. Cerebral blood flow velocity in the human newborn: the value of its determination. Pediatrics 1982;70:147-52.

31. Martin CG, Snider AR, Katz SM, Peabody JL, Brady JP. Abnormal cerebral blood flow patterns in preterm infants with a large patent ductus arteriosus. J Pediatr 1982;101:587-93.

32. Takahashi S, Kakiuchi S, Nanba Y, Tsukamoto K, Nakamura T, Ito Y. The perfusion index derived from a pulse oximeter for predicting low superior vena cava flow in very low birth weight infants. J Perinatol 2010;30:265-9.

33. Prietsch V, Maier R, Schmitz L, Obladen M. Long-term variability of heart rate increases with successful closure of patent ductus arteriosus in preterm infants. Biol Neonate 1992;61:142-149.

34. Patrick J McNamara AS 2007 Towards rational management of the patent ductus arteriosus: the need for disease staging. Arch Dis Child Fetal Neonatal Ed. 92:F424-F427. 